\title{
A strategy to minimize the sensing voltage drift error in a transistor biosensor with a nanoscale sensing gate
}

\author{
Hyun Woo Son $1, *$ \\ Minhong Jeun ${ }^{1, *}$ \\ Jaewon Choi ${ }^{1,2}$ \\ Kwan Hyi Lee ${ }^{1,2}$
}

'Center for Biomaterials, Biomedical Research Institute, Korea Institute of Science and Technology, Seoul, ${ }^{2}$ Department of Biomedical Engineering, Korea University of Science and Technology, Daejeon, Republic of Korea

*These authors contributed equally to this work
Correspondence: Kwan Hyi Lee Center for Biomaterials, Biomedical Research Institute, Korea Institute of Science and Technology, 5 Hwarangno 14-gil, Seongbuk-gu, Seoul 02792, Republic of Korea $\mathrm{Tel}+8229586804$

Fax +82 29585308

Email kwanhyi@kist.re.kr
This article was published in the following Dove Press journal:

International Journal of Nanomedicine

II April 2017

Number of times this article has been viewed

Abstract: An ion-sensitive field-effect transistor (ISFET) biosensor is thought to be the center of the next era of health diagnosis. However, questions are raised about its functions and reliability in liquid samples. Consequently, real-life clinical applications are few in number. In this study, we report a strategy to minimize the sensing signal drift error during bioanalyte detection in an ISFET biosensor. A nanoscale $\mathrm{SnO}_{2}$ thin film is used as a gate oxide layer (GOL), and the surface of the GOL is chemically modified for improving bioanalyte-specific binding and for reducing undesirable ion reactions in sample solutions. The ISFET biosensor with surfacemodified GOL shows significantly reduced sensing signal error compared with an ISFET with bare GOL in both diluted and undiluted phosphate buffered saline solutions.

Keywords: extended gate, surface treatment, biosensor, $\mathrm{SnO}_{2}$, ISFET

\section{Introduction}

The pH-sensing ion-sensitive field-effect transistor (ISFET), first proposed by Bergveld in 1970, has become the basis for many contemporary semiconductors and their respective biosensors. ${ }^{1}$ In an ISFET, the ions within the sample media undergo multiple environment-influenced reactions. The resulting molecules from these reactions accumulate upon the gate oxide layer (GOL), and the resulting change in charge affects the conductance in the ISFET channel., ${ }^{2,3}$ Consequently, the conductance between the source and the drain will differ, and this change is expressed as an electrical signal.

The GOLs of ISFETs are usually made up of dielectric materials, such as $\mathrm{SnO}_{2},{ }^{4-7}$ $\mathrm{ZnO},{ }^{8} \mathrm{SiO}_{2},{ }^{9,10} \mathrm{Al}_{2} \mathrm{O}_{3},{ }^{11} \mathrm{Si}_{3} \mathrm{~N}_{4},{ }^{12,13} \mathrm{Ta}_{2} \mathrm{O}_{5},{ }^{14} \mathrm{TiO}_{2},{ }^{15}$ and $\mathrm{WO}_{3},{ }^{16}$ which readily react with the $\mathrm{H}^{+}$and $\mathrm{OH}^{-}$of the media. Dielectric materials that have a high reactivity with ions are used as either $\mathrm{pH}$ sensor or gas sensor. ${ }^{17}$ However, because other ions within the sample media also affect the surface charge on the GOL, the electrical characteristics such as the threshold voltage and channel conductance will be influenced.

ISFET-based biosensors, which detect bioanalytes, are affected by the undesirable ions in the media, and the resulting fluctuations or noise may cause the results to be deemed untrustworthy. Although the signal may stabilize as time progresses and the reaction becomes saturated (reaches equilibrium state), the noise factor must be acknowledged when evaluating the final signal. Because the target bioanalyte is already in small amounts inside the target solution, any other factors that may affect the signal, such as the ions within the solution media, will affect the sensitivity of the biosensor. When the biosensor applies highly reactive nanowires and thin films 
to enhance its sensitivity, the unwanted noise factor may also be increased and affect the result even more.

In this study, we report a strategy to minimize the sensing voltage error in the ISFET biosensor. To improve specific bioanalyte binding and to reduce undesirable ion reactions, an optimal presurface treatment on the gate oxide is demonstrated. In addition, the sensing voltage drift error $\left(\Delta V_{\mathrm{df}}\right)$ of the ISFET biosensor before presurface treatment and after presurface treatment is analyzed. Finally, because multiple teams that study and utilize the ISFET biosensor dilute the media to obtain a longer Debye screening length, we analyzed the $\Delta V_{\mathrm{df}}$ for $1 \times$ phosphate buffered saline (PBS) and $0.01 \times$ PBS solutions.

\section{Experimental}

\section{Sensing gate fabrication}

The GOL of an ISFET biosensor was fabricated by depositing an $\mathrm{SnO}_{2}(99.9 \%)$ thin film on the top of an indium tin oxide (ITO) glass (ITO thickness: $300 \mathrm{~nm}$ ). The $80 \mathrm{~nm}$ $\mathrm{SnO}_{2}$ film was deposited using a radio frequency (RF) magnetron sputtering system (RF power: $50 \mathrm{~W}$, base pressure: $2 \times 10^{-6}$ Torr, work pressure: 18 mTorr, Ar gas flow: $5 \mathrm{sccm}$ ). The polydimethylsiloxane (PDMS) block was manufactured from a Sylgard 184 Silicone Elastomer Kit (Dow Corning, Seoul, Republic of Korea), which was composed of a base and a curing agent. The base and curing agent were mixed at a 10:1 ratio and baked at $60^{\circ} \mathrm{C}$ for $4 \mathrm{~h}$. The PDMS block was then punctured with $6 \mathrm{~mm}$ holes to form a sample media reservoir. To attach the PDMS to the surface of GOL, the GOL was sonicated in deionized water for $1 \mathrm{~min}$ and then sonicated in ethanol for $1 \mathrm{~min}$. The GOL was then subjected to $\mathrm{N}_{2}$ gas blowing. The PDMS block and GOL were then placed inside an $\mathrm{O}_{2}$ plasma system (CUTE-MPR; Femto Science, Gyeonggi-Do, Republic of Korea) and treated with $\mathrm{O}_{2}$ plasma (DC power: $70 \mathrm{~W}$, time: $1 \mathrm{~min}, \mathrm{O}_{2}$ flow: $30 \mathrm{sccm}$, base pressure: $5 \times 10^{-2}$ Torr, working pressure: $5 \times 10^{-1}$ Torr). The PDMS block and GOL were then attached together to form the sensing gate (Figure 1).

\section{Presurface treatment of GOL and antibody fixation}

The GOL surface of the sensing gate was treated with plasma to form $\mathrm{OH}$ functional groups on the reservoir surface. Then, 5\% 3-aminopropyltriethoxysilane (APTES) was prepared and quickly added to the surface of the GOL for the formation of $\mathrm{NH}_{2}$ functional groups. After the addition of the APTES, the GOL was placed inside a petri dish and the petri dish was sealed with parafilm for $1 \mathrm{~h}$. The chemical reaction should occur in a dark place. After $1 \mathrm{~h}$, the sensing gate was sonicated in ethanol for $1 \mathrm{~min}$ and dried with $\mathrm{N}_{2}$ gas. The sensing gate was baked on a hot plate at $120^{\circ} \mathrm{C}$.

To functionalize the GOL surface of the sensing gate with $\mathrm{COOH}$ functional groups, 5\% $1 \mathrm{M}$ succinic anhydride was made from succinic anhydride powder and dimethyl formamide (DMF) and added onto the surface of the GOL. The petri dish was sealed with parafilm and placed inside an incubator at $37^{\circ} \mathrm{C}$ for overnight. The presurface-treated sensing gate was washed with DMF and deionized water for 20 s. This GOL was then treated with 1-ethyl-3(3-dimethylaminopropyl) carbodiimide (EDC) HCL and

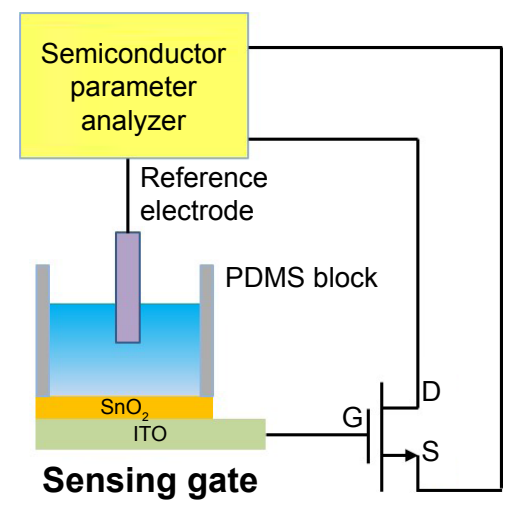

Presurface treatment and blocking

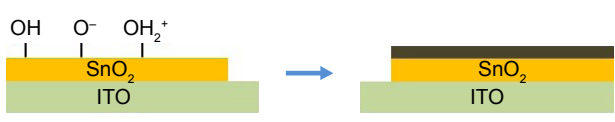

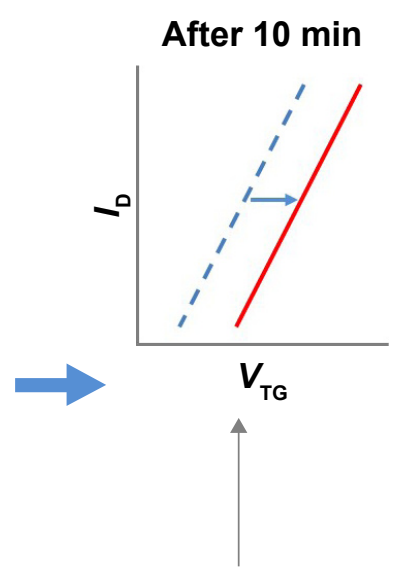

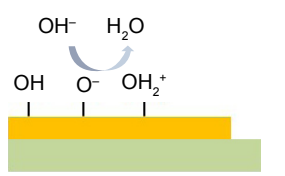

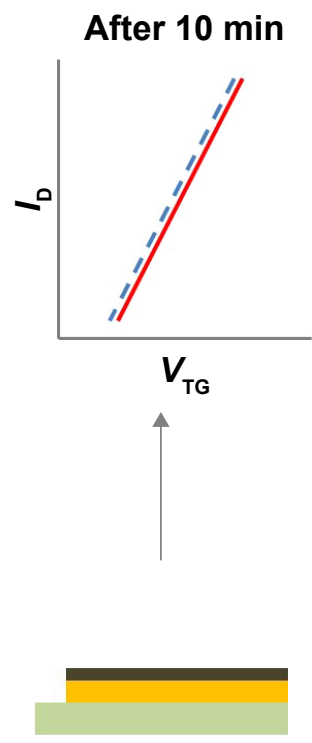

Figure I ISFET $I_{D}-V_{T G}$ measurement configuration and sensing gates with bare $\mathrm{SnO}_{2}$ thin film and presurface-treated $\mathrm{SnO}_{2}$ thin film. Abbreviations: ISFET, ion-sensitive field-effect transistor; ITO, indium tin oxide; PDMS, polydimethylsiloxane; G, gate; D, drain; S, source. 
Sulfo- $N$-hydroxysulfosuccinimide (NHS) chemistry. ${ }^{18}$ GOL after the addition of EDC and Sulfo-NHS was identified as a surface-treated GOL (ST-GOL). Antibodies were then added to the wells. Here, we used prostate-specific membrane antigen (PSMA) antibodies. The PSMA antibody was provided by "Biorbyt" (catalog number orb25888; San Francisco, CA, USA). The host of the PSMA antibody was mouse. It had an IgG2b isotype structure and was specific to the c-terminal of a PSMA antigen. The PSMA antibody was conjugated onto the sensing gate surface at a concentration of $100 \mathrm{nM}$.

To prevent unwanted reactions with unreacted chemical cross-linkers left on the surface, $1 \mathrm{M}$ ethanolamine ( $\mathrm{pH} 8.5$ ) was added. After adding the ethanolamine to the ST-GOL, the ST-GOL was washed with $1 \times$ PBS. In order to protect the ST-GOL from nonspecific binding, 10\% bovine serum albumin (BSA) was added and left to undergo the chemical reaction for $1 \mathrm{~h}$. Finally, the ST-GOL was washed with $1 \times$ PBS (Figure 2).

\section{Measurement}

A commercial $\mathrm{Ag} / \mathrm{AgCl}$ reference electrode (Qrins, MF-2097), a commercial CMOS transistor (ON Semiconductor, MC14007UB, N-type MOSFET), and a high precision semiconductor parameter analyzer (4200-SCS; Keysight Technologies, Santa Rosa, CA, USA) were utilized for the measurement of the $\Delta V_{\mathrm{df}}$.

The experiment was conducted under different conditions. In the initial condition, the $\Delta V_{\mathrm{dr}}$ of the $I-V$ curves was measured at the $0,1,3$, and 5 min marks after inserting a $0.01 \times$ PBS solution into PDMS reservoirs on all the sensing gates. In the second condition, the $0.01 \times$ PBS was replaced with $1 \times$ PBS and the solution was added into the PDMS reservoirs on all the sensing gates. The measurement was taken at the
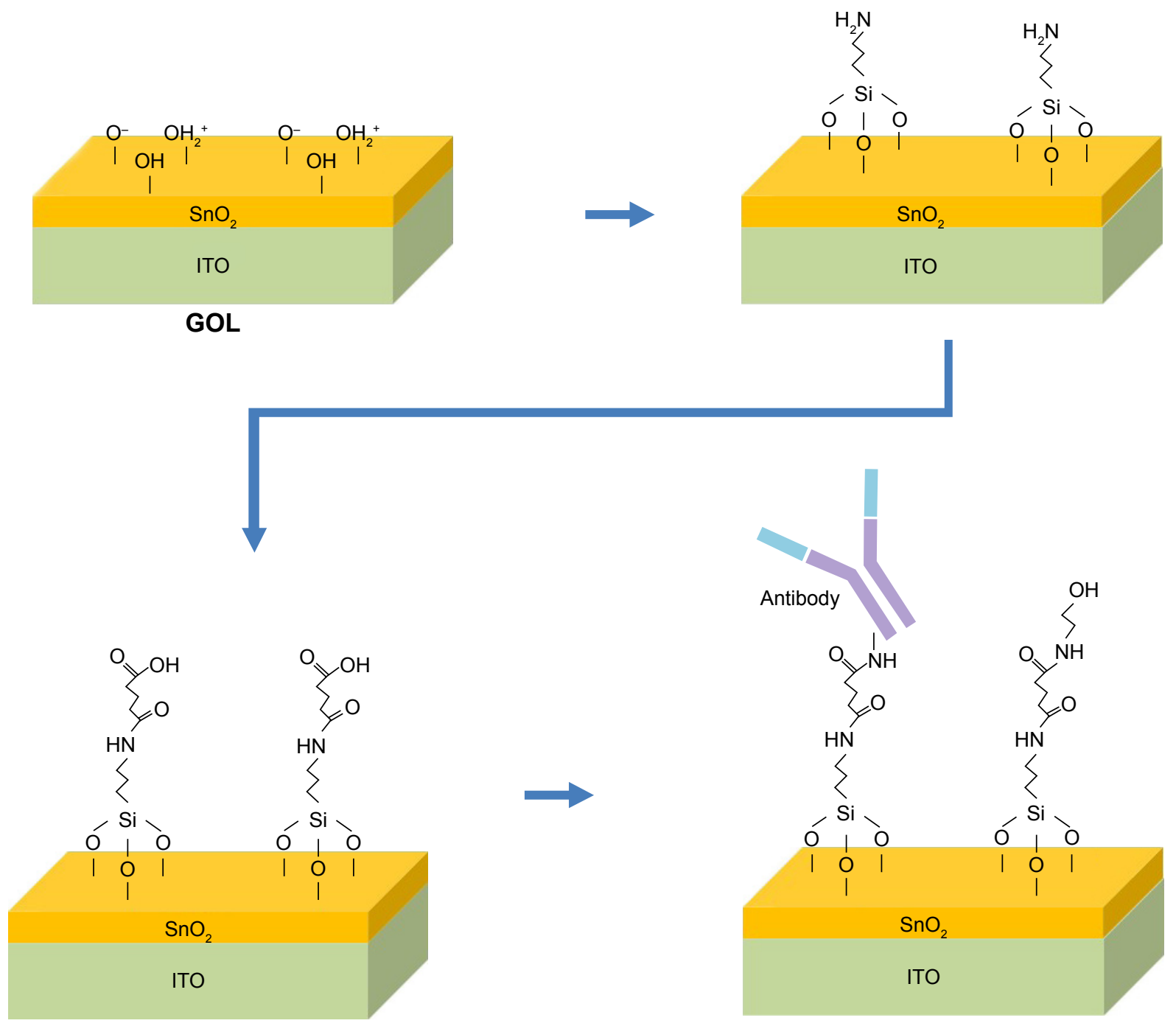

Figure 2 Presurface treatment procedure of $\mathrm{SnO}_{2} \mathrm{GOL}$ and antibody conjugation with EDC-Sulfo-NHS chemistry.

Abbreviations: EDC, I-ethyl-3-(3-dimethylaminopropyl) carbodiimide; GOL, gate oxide layer; ITO, indium tin oxide; NHS, N-hydroxysulfosuccinimide. 
same time points as the initial condition $(0,1,3$, and 5 min marks). The chemical compositions of the PBS solution are as follows: 1) potassium phosphate monobasic $\left(\mathrm{KH}_{2} \mathrm{PO}_{4}\right)$ $1.0589 \mathrm{mM}, 2)$ sodium chloride $(\mathrm{NaCl}) 155.172 \mathrm{mM}$, and 3) sodium phosphate dibasic $\left(\mathrm{Na}_{2} \mathrm{HPO}_{4} \cdot 7 \mathrm{H}_{2} \mathrm{O}\right) 2.9665 \mathrm{mM}$. There is no calcium chloride or magnesium chloride in this buffer. The $\mathrm{pH}$ of the PBS is 7.4.

In addition, to measure the $\Delta V_{\mathrm{df}}$ difference between the different surface treatments of the GOL, $1 \times$ PBS was added to bare GOL, ST-GOL without antibodies, and ST-GOL with antibodies. The $\Delta V_{\mathrm{dr}}$ was measured at the $0,1,3,5$, and 10 min marks (Figure 1).

\section{Results and discussion}

We investigated the $\Delta V_{\mathrm{df}}$ of a bare GOL, an ST-GOL without antibodies, and an ST-GOL with antibodies in $0.01 \times$ PBS (Figure $3 \mathrm{~A}-\mathrm{C}$ ). After adding $0.01 \times$ PBS into all respective PDMS reservoirs of the GOL and waiting for $1 \mathrm{~min}$, the voltage values were measured. Subsequent analyses were conducted in 2 min intervals and repeated two times to identify the $\Delta V_{\mathrm{df}}$. In the case of the bare GOL, a large $\Delta V_{\mathrm{df}}$ of $21.5 \mathrm{mV}$ for $5 \mathrm{~min}(4.3 \mathrm{mV} / \mathrm{min})$ was observed. This error value reaches $36.3 \%$ of an FET's Nernst limit $(59.3 \mathrm{mV} / \mathrm{pH}) .{ }^{19,20}$ This result implies that in a situation where sensing is conducted in a low antigen concentration range, which is lower than the ionic concentration change range following a change in $1 \mathrm{pH}$, it is hard to obtain a trustworthy sensing signal.

An additional phenomenon that was observed was the consistent shift of the $\Delta V_{\mathrm{df}}$ as time passes. This is because the nonpreconditioned GOL has an unstable surface and, thus, reacts with numerous ions of the $\mathrm{PBS}\left(\mathrm{OH}^{-}, \mathrm{H}^{+}\right.$, and $\left.\mathrm{O}_{2}^{-}\right)$, for a long time. This phenomenon contributes to sensors being unable to produce reliable results when exposed to extended periods of testing.

In the cases of the ST-GOL without antibodies and the ST-GOL with antibodies, the $\Delta V_{\mathrm{df}}$ values were significantly reduced. The ST-GOL without antibodies showed a $\Delta V_{\mathrm{df}}$ of $11.37 \mathrm{mV} / 5 \mathrm{~min}(2.3 \mathrm{mV} / \mathrm{min})$, and the ST-GOL with

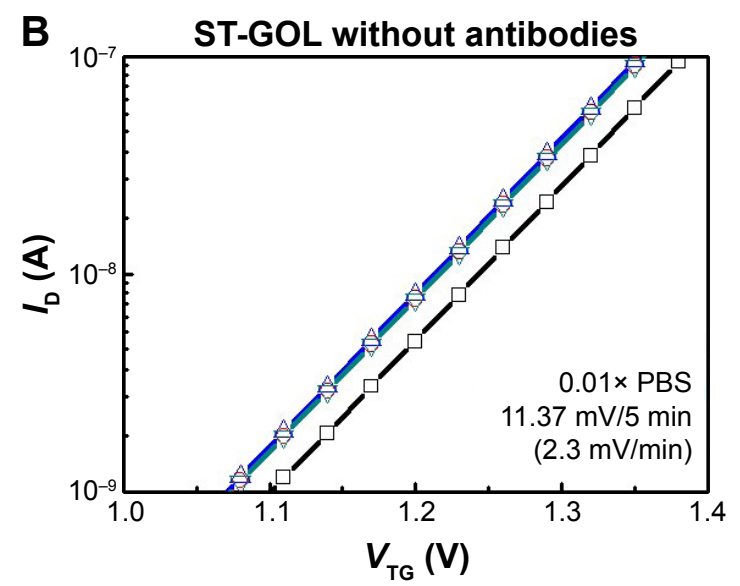

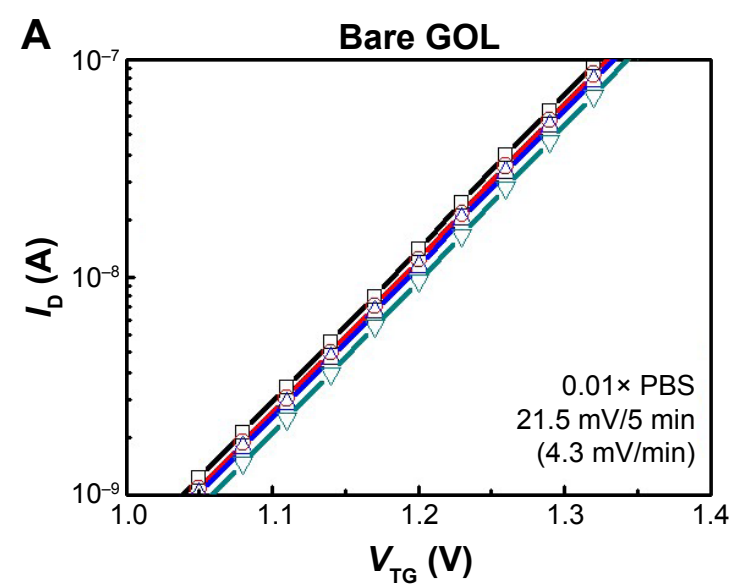

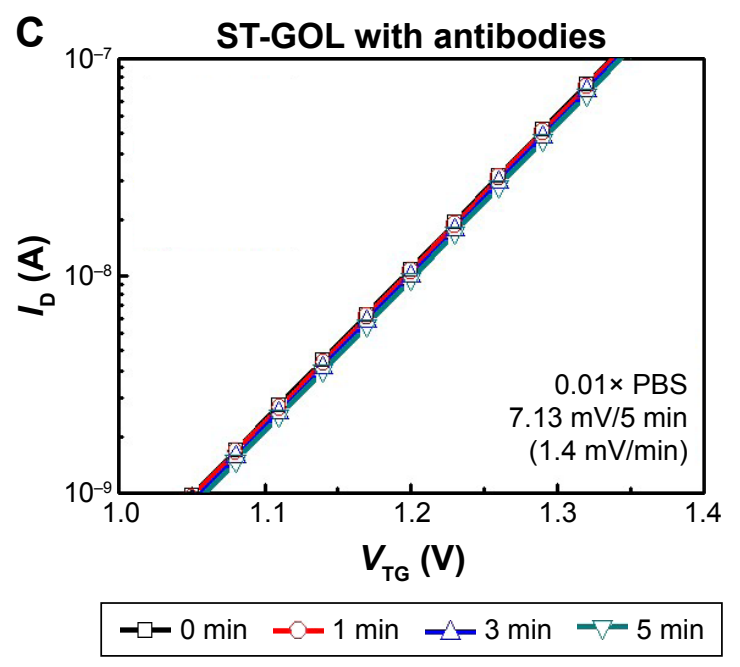

Figure 3 Voltage drift error $\left(\Delta V_{\mathrm{df}}\right)$ of $(\mathbf{A})$ a bare GOL, (B) a presurface-treated GOL without antibodies, and $(\mathbf{C})$ a presurface-treated GOL with antibodies in 0.0 I $\times$ PBS solution.

Abbreviations: GOL, gate oxide layer; ST-GOL, surface-treated GOL; PBS, phosphate buffered saline. 

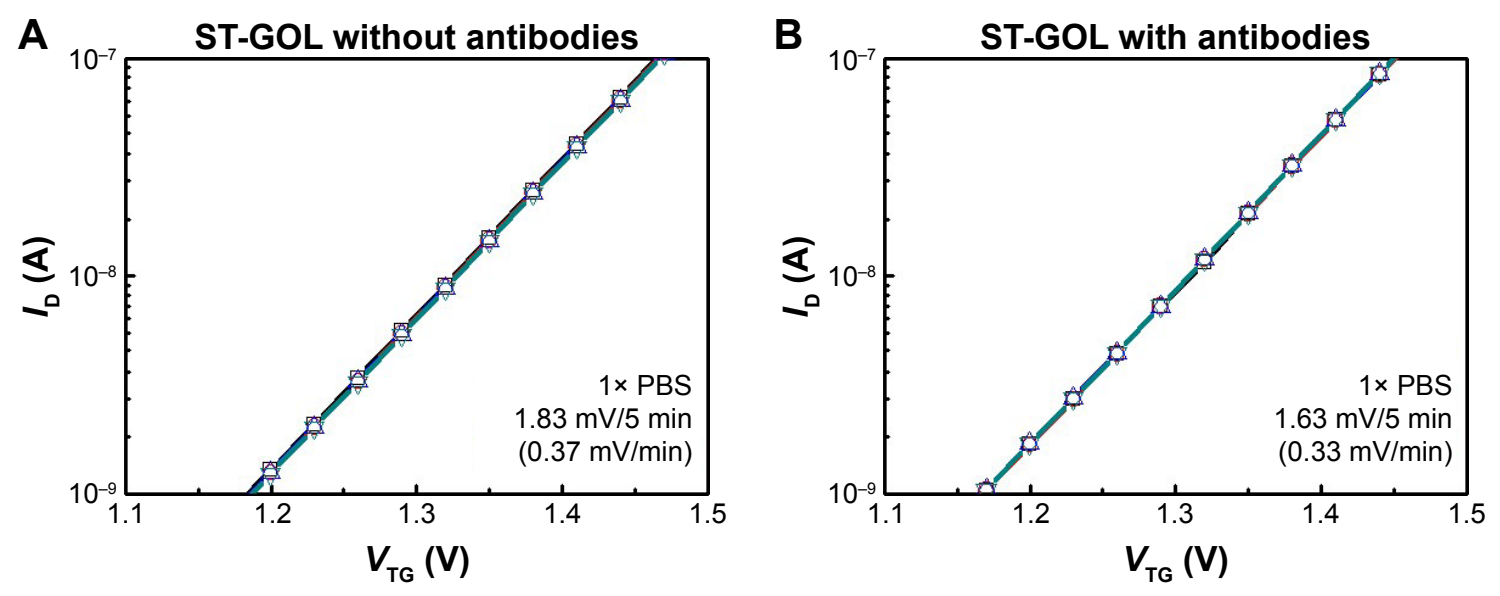

$\neg-0 \min \rightarrow 1 \min \rightarrow-\hookrightarrow \min \rightarrow-5 \min$

Figure 4 Voltage drift error $\left(\Delta V_{\mathrm{df}}\right)$ of $(\mathbf{A})$ a presurface-treated gate oxide layer without antibodies and $(\mathbf{B})$ a presurface-treated gate oxide layer with antibodies measured in the undiluted $\mathrm{I} \times \mathrm{PBS}$ solution.

Abbreviations: ST-GOL, surface-treated gate oxide layer; PBS, phosphate buffered saline.

antibodies showed a $\Delta V_{\mathrm{df}} 7.13 \mathrm{mV} / 5 \mathrm{~min}(1.4 \mathrm{mV} / \mathrm{min})$. These values are only $19.2 \%$ of the Nernst limit and $12 \%$ of the Nernst limit, respectively. The reasons for the decreased $\Delta V_{\text {df }}$ can be thought to be due to the following. First, the random chemical reactions between the chemical links on the surface of GOL and ions of the PBS were restricted by the presurface treatment. Second, the number of residual chemical links that can react and bind to the ions within the PBS is limited and this causes saturation to occur very quickly.

Figure $4 \mathrm{~A}$ and B shows the $\Delta V_{\text {df }}$ values of adding undiluted $1 \times$ PBS solution to an ST-GOL without antibodies and to an ST-GOL with antibodies. The experiment was conducted under the same conditions as the experiment of Figure 3. As can be seen from the results, the $\Delta V_{\text {df }}$ results are much smaller than the $\Delta V_{\text {df }}$ of its $0.01 \times$ PBS counterpart: the ST-GOL without antibodies has a $\Delta V_{\text {df }}$ of $0.37 \mathrm{mV} / \mathrm{min}$ and the ST-GOL with antibodies has a $\Delta V_{\mathrm{df}}$ of $0.33 \mathrm{mV} / \mathrm{min}$. The significant reduction of the $\Delta V_{\text {df }}$ values is because of the Debye screening length in the $1 \times$ PBS. The $1 \times$ PBS has the much smaller Debye screening length of $0.7 \mathrm{~nm}$ than $0.01 \times$ PBS's Debye screening length of $\sim 7.3 \mathrm{~nm}^{21,22}$ Consequentially, the GOL can only detect much smaller ions in $1 \times \mathrm{PBS}$, and as a result, the chemical reaction is rapidly saturated (or stabilized).

Figure 5 shows the results for all GOL $\Delta V_{\mathrm{df}}$ values that were measured at the $0,1,3,5,10,15$, and $20 \mathrm{~min}$ marks in $1 \times$ PBS. For the bare GOL, the chemical links of the $\mathrm{SnO}_{2}$ surface reacted continuously with the ions in the PBS and the $\Delta V_{\mathrm{df}}$ increased as time passed. This result is consistent with our past experiment. However, GOL that has undergone presurface treatment showed complete saturation by the ions in $1 \times$ PBS by the 1 min mark. Consequently, a presurface-treated GOL utilizing $1 \times$ PBS can decrease the noise from unwanted ions and produce a reliable biosensing signal.

The results clearly indicate that in scenarios where the ISFET is used to sense biomarkers, the undiluted $1 \times$ PBS could produce trustworthy biosensing signals. However, the $1 \times$ PBS will lead to a much shorter Debye screening length and subsequent biomarker detection limit. This can lead to problems if a lengthy or bulky bioconjugate, such as whole antibodies, is utilized. To solve the problem from the short Debye screening length, small capture linkers, such as antibody fragments, aptamers, and peptides, could be used instead of large whole antibodies.

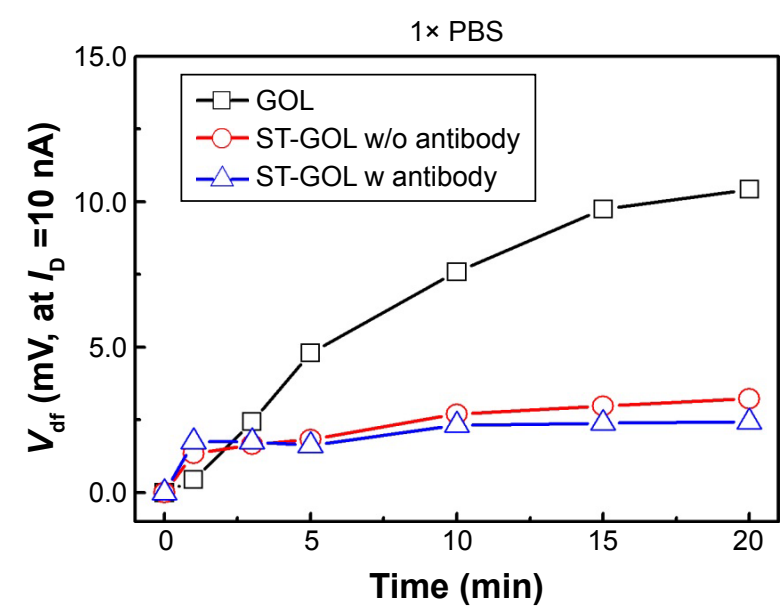

Figure 5 Results of voltage drift error $\left(\Delta V_{d f}\right)$ values for all the GOLs measured at the $0,1,3,5,10,15$, and 20 min marks in IXPBS solution.

Abbreviations: GOLs, gate oxide layers; ST-GOL, surface-treated GOL; PBS, phosphate buffered saline; w, with; w/o, without. 


\section{Conclusion}

This study used $\mathrm{SnO}_{2}$ as a gate oxide sensing layer in an ISFET biosensor to capture biomarkers and analyze their biosignals. The results demonstrate that proper surface treatment of a gate oxide sensing layer in an ISFET can significantly reduce the sensing signal error. Because the amounts of biomarkers in samples are usually minuscule, a biosensor must be sensitive enough to detect these biomarkers at low concentrations. To produce a longer Debye screening length for the ISFET biosensor, the sample media, which were the PBS solutions in this study, are diluted. However, this could lead to a situation where the media itself can cause both a large $\Delta V_{\mathrm{df}}$ and a large margin of error, thus making the resulting biosensing signal unreliable. To decrease such error from the media, a more concentrated medium, such as $1 \times$ PBS, is utilized. The $1 \times$ PBS medium quickly reacts and saturates the surface of sensing gate and subsequently stabilizes the electrical potential. An unfortunate side result from adding the concentrated medium is the shortening of the Debye screening length. To overcome this obstacle, aptamers, peptides, and antibody fragments and immobilization materials that are shorter than a whole antibody need to be utilized to maintain a reliable sensor sensitivity outcome.

\section{Acknowledgment}

This study was supported by the Bio \& Medical Technology Development Program of the NRF funded by the Korean government MSIP (2015M3A9E2029265) and Korea Health Technology R\&D Project through the Korea Health Industry Development Institute (KHIDI) funded by the Ministry of Health \& Welfare, Republic of Korea (HI15C3078-020015).

\section{Disclosure}

The authors report no conflicts of interest in this work.

\section{References}

1. Bergveld P. Development of an ion-sensitive solid-state device for neurophysiological measurements. IEEE Trans Biomed Eng. 1970; 17(1):70-71.
2. Pablo Diniz B. An embedded measurement system for the electrical characterization of EGFET as a pH sensor. Meas Sci Technol. 2014; 25(2):027001.

3. Lee C-S, Kim KS, Kim M. Ion-sensitive field-effect transistor for biological sensing. Sensors. 2009;9(9):7111-7131.

4. Jung-Chuan C, Pik-Kwan $\mathrm{K}$, Zhi-Jie C. $\mathrm{SnO}_{2}$ separative structure extended gate $\mathrm{H}^{+}$-ion sensitive field effect transistor by the sol-gel technology and the readout circuit developed by source follower. Jpn J Appl Phys. 2003;42(11):6790-6794.

5. Liao H-K, Chou J-C, Chung W-Y, Sun T-P, Hsiung S-K. Study of amorphous tin oxide thin films for ISFET applications. Sens Actuators B Chem. 1998;50(2):104-109.

6. Jang H-J, Ahn J, Kim M-G, et al. Electrical signaling of enzyme-linked immunosorbent assays with an ion-sensitive field-effect transistor. Biosens Bioelectron. 2015;64:318-323.

7. Lee IK, Jeun M, Jang HJ, Cho WJ, Lee KH. A self-amplified transistor immunosensor under dual gate operation: highly sensitive detection of hepatitis B surface antigen. Nanoscale. 2015;7(40):16789-16797.

8. Batista PD, Mulato M. ZnO extended-gate field-effect transistors as pH sensors. Appl Phys Lett. 2005;87(14):143508.

9. Leistiko $O$. The selectivity and temperature characteristics of ion sensitive field effect transistors. Phys Scr. 1978;18(6):445-450.

10. Schöning MJ, Tsarouchas D, Beckers L, et al. A highly long-term stable silicon-based $\mathrm{pH}$ sensor fabricated by pulsed laser deposition technique. Sens Actuators B Chem. 1996;35(1):228-233.

11. Bousse $\mathrm{L}$, van den Vlekkert $\mathrm{HH}$, de Rooij NF. Hysteresis in $\mathrm{Al}_{2} \mathrm{O}_{3}$-gate ISFETs. Sens Actuators B Chem. 1990;2(2):103-110.

12. Liu BD, Su YK, Chen SC. Ion-sensitive field-effect transistor with silicon nitride gate for $\mathrm{pH}$ sensing. Int J Electron. 1989;67(1):59-63.

13. Rocher V, Poyard S, Jaffrezic-Renault N, Ajoux C, Lemiti M, Sibai A. Photo-CVD silicon nitride thin layers as $\mathrm{pH}-\mathrm{ISFET}$ sensitive membrane. Sens Actuators B Chem. 1994;19(1):342-347.

14. Poghossian AS. The super-Nernstian $\mathrm{pH}$ sensitivity of $\mathrm{Ta}_{2} \mathrm{O}_{5}$-gate ISFETs. Sens Actuators B Chem. 1992;7(1):367-370.

15. Jung Chuan C, Lan Pin L. Study of $\mathrm{TiO}_{2}$ thin films for ion sensitive field effect transistor application with RF sputtering deposition. Jpn J Appl Phys. 2004;43(1):61-65.

16. Chou JC, Chiang JL. Study on the amorphous tungsten trioxide ionsensitive field effect transistor. Sens Actuators B Chem. 2000;66(1-3): 106-108.

17. Watson J, Ihokura K. Gas-sensing materials. MRS Bull. 1999; 24(6):14-17.

18. Sohn Y-S, Lee YK. Site-directed immobilization of antibody using EDC-NHS-activated protein A on a bimetallic-based surface plasmon resonance chip. J Biomed Opt. 2014;19(5):051209.

19. Sarkar D, Liu W, Xie X, Anselmo AC, Mitragotri S, Banerjee K. $\mathrm{MoS}_{2}$ field-effect transistor for next-generation label-free biosensors. ACS Nano. 2014;8(4):3992-4003.

20. Knopfmacher O, Tarasov A, Fu W, et al. Nernst limit in dual-gated Si-nanowire FET sensors. Nano Lett. 2010;10(6):2268-2274.

21. Elnathan R, Kwiat M, Pevzner A, et al. Biorecognition layer engineering: overcoming screening limitations of nanowire-based FET devices. Nano Lett. 2012;12(10):5245-5254.

22. Stern E, Wagner R, Sigworth FJ, Breaker R, Fahmy TM, Reed MA. Importance of the Debye screening length on nanowire field effect transistor sensors. Nano Lett. 2007;7(11):3405-3409.
International Journal of Nanomedicine

\section{Publish your work in this journal}

The International Journal of Nanomedicine is an international, peerreviewed journal focusing on the application of nanotechnology in diagnostics, therapeutics, and drug delivery systems throughout the biomedical field. This journal is indexed on PubMed Central, MedLine, CAS, SciSearch ${ }^{\circledR}$, Current Contents ${ }^{\circledR} /$ Clinical Medicine,

\section{Dovepress}

Journal Citation Reports/Science Edition, EMBase, Scopus and the Elsevier Bibliographic databases. The manuscript management system is completely online and includes a very quick and fair peer-review system, which is all easy to use. Visit http://www.dovepress.com/ testimonials.php to read real quotes from published authors. 